\title{
Outdoor Autonomous Landing of a Quadcopter on a Moving Platform using Off-board Computer Vision
}

\author{
G.L. Goh, G.D. Goh, Z.W. Zhong* \\ School of Mechanical and Aerospace Engineering, Nanyang Technological University, 50 Nanyang Avenue, \\ Singapore 639798, Republic of Singapore \\ E-mail: mzwzhong@ntu.edu.sg (Corresponding author)
}

Received: 28 September 2019; Accepted: 23 October 2019; Available online: 30 November 2019

\begin{abstract}
This paper presents a method that enables a quadcopter to perform autonomous landing on a moving platform using computer vision. In addition, the system implementation of the computer vision technique is presented. Unlike other researches, the camera is mounted on the moving platform instead of being installed on the quadcopter. Besides, the computer vision system is tested outdoor, and the results such as the performance and the accuracy are presented. In the stationary platform test, 5 out of 10 landings fall within $30 \mathrm{~cm}$ from the center. In the moving platform test, the maximum platform-moving speed for autonomous landing is $2 \mathrm{~m} / \mathrm{s}$. Hence, it is proven that this methodology is feasible. Lastly, the advantages and limitations of the computer vision technique proposed are discussed.
\end{abstract}

Keywords: Autonomous landing; Camera; Computer vision; Moving platform; Quadcopter; Unmanned aerial vehicle.

\section{Introduction}

Unmanned aerial vehicles (UAVs) are flying machines that do not require human operators on board during the missions [1, 2]. UAVs can either be piloted remotely or fly autonomously depending on the mission's objectives and requirements.

The development of UAVs occurred rapidly during World War 1 and World War 2. This was due to the ability of UAVs to allow real-time intelligence, surveillance and reconnaissance information from the battlefield without risking lives of pilots [3]. Apart from that, a UAV is much lighter and it helps in reducing the use of fuel for the mission, which increases both range and flight endurance of the craft. Hence, it is easy to understand the importance of the role of UAVs in military and the reason for UAVs getting more attention worldwide.

Besides the use of UAVs in military operations, they are also getting more attention from many other fields such as civil, commercial, agriculture and multimedia, for which UAVs are used for security, surveillance, search and rescue purposes [1]. The use of UAVs is not restricted to the few examples mentioned above; in the US, UAVs are used to collect meteorological and ecological data. Hence, UAVs are seen to have great potentials in nonmilitary purposes due to their versatility. For this reason, a lot of research has been carried out to explore creative and novel designs of UAVs for different purposes.

Nowadays, UAVs no longer take only the form of conventional fixed wing aircraft, but also rotary wing, glider, and gyroplane forms [4]. In recent years, multi-rotors have gained much attention from hobbyists, the public as well as researchers. This is probably due to the unique flight characteristics of multi-rotors, which are easy to control and capable of hovering, flying at low speeds and lifting heavy payloads. However, it is interesting to note that a multi-rotor is a statically unstable system, which means that it requires a flight controller unit to make it flyable [5]. With the introduction of small and cheap flight controller units in the market, it is no wonder that multirotors are getting more affordable and well received by the public.

Of all the multi-rotors, the quadcopter is the more popular choice among aviation hobbyists and researchers as compared to other multi-rotors. The quadcopter is a type of multi-rotors with 4 rotary wings to produce sufficient lift for various maneuvers [5, 6]. With the flight controller unit, the quadcopter can be controlled manually or programmed to fly autonomously for various missions such as flying to pre-programmed waypoints.

A lot of research has been carried out to explore the usefulness of multi-rotors such as fire-fighting UAVs, reconnaissance UAVs, morphing UAVs and more [7-9]. Interestingly, there is also a noticeable trend that more researchers are shifting their research focuses towards the development of autonomous UAVs. Among the autonomous UAV researches, the developments of swarming algorithms [10] for multiple UAVs, and flight control algorithms for multi-rotors are the more popular research fields [11, 12]. However, there is less attention on the 
development of the autonomous landing of UAVs. After doing literatures review, it is found that there are only limited papers and reports that have discussed about this research area. Hence, it is worthwhile to further investigate the feasibility of the autonomous landing.

Position sensing is a very important aspect in the control of UAVs. There are many position sensing technologies available in the market. In most of the researches, the motion capture technology is widely used as it can provide more accurate and reliable data as compared to other technologies. It is proven by many researchers that the UAVs are able to make very precise and high agility maneuvers using the motion capture technology [13, 14]. Besides, the motion capture technology also allows the study of multi-UAV interactions, as the technology is able to detect multiple UAVs at the same time [13]. However, this technology is only suitable for indoor flight testing and not practical for outdoor flight testing purposes. Hence, an alternative position sensing technology is needed if the flight test is to be carried out outdoor.

There are several approaches used by groups of researchers to carry out the landing maneuver. Some of them use an optical flow sensor to give information about the altitude of the UAV [15], whereas the others use a camera to give information about the position and altitude of the UAV [16]. In case of using a camera as a sensor, a landing target is needed as a reference for the UAV. There are several landing targets that have been tested by researchers such as an H-shaped landing target [17] and a landing target with alternate black and white circles [16]. The researchers claimed that the system accuracy of $5 \mathrm{~cm}$ in translation is achievable. There are also studies that have shown autonomous landing of UAV without using landing site identification [16]. However, these landing maneuvers are carried out using a stationary landing platform.

In contrast, there is relatively less research that has been done to investigate the autonomous landing of a UAV on a moving platform. In one of the research, a helicopter is used as a testbed to validate a vision-based algorithm, which enables the helicopter to identify, track and land on a moving platform using vision and GPS [17]. In another study, an X-3D-BL quadcopter is used as a testbed; however, the research failed to develop an autonomous landing controller due to the ground effect [14]. Other than that, some researchers also tested image-based visual servoing technique to track a moving platform and land the quadcopter on it [18]. However, none of the studies mentioned above is carried out outdoor.

Nevertheless, there was a research group that carried out the experiment outdoor [19]. The research team attached an omnidirectional camera and a smart phone on the quadcopter for data acquisition and image processing respectively. They integrated a nonlinear estimation model, geolocation filter and flight controller into a flight system. According to the flight test result, the quadcopter successfully landed on the moving platform using the developed system. However, it is found that the moving platform is moving at a very low speed $(0.3 \mathrm{~m} / \mathrm{s})$, which makes it impractical to be used for real-life applications.

As discussed above, there is less attention on the research relating to the implementation of an autonomous landing system for a mobile base. It is necessary to carry out research on designing and controlling of a quadcopter to perform such landing maneuver. Therefore, this work focuses on developing a reliable and robust technique that can be implemented on a quadcopter to perform autonomous landing on a moving platform at a speed of $2 \mathrm{~m} / \mathrm{s}$ tested outdoor.

\section{Dynamic model of quadcopter}

\subsection{Coordinate systems}

In order to fully describe the motion of the quadcopter, a ground reference frame and a local/body reference frame are required. In this work, the local and global reference frames shown in Fig. 1 are used.

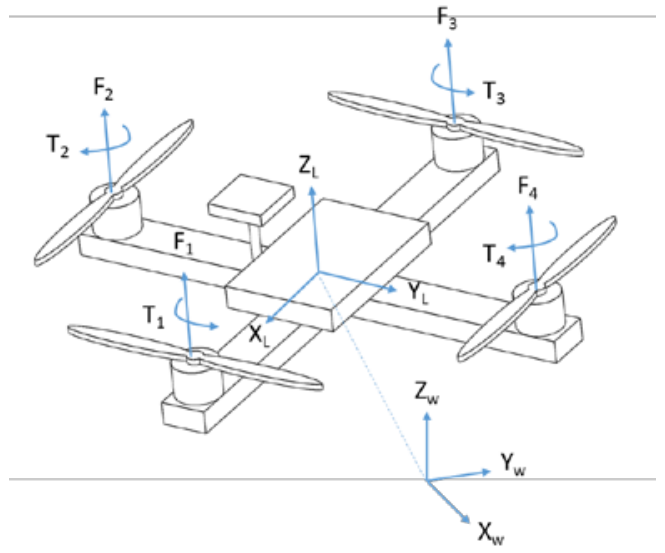

Figure 1. Dynamics of the quadcopter and coordinate systems. 
The motion and attitude of the quadcopter can be described using the 12 parameters, which can be obtained from the reference systems. For example, the position $\left[\begin{array}{lll}x & y & z\end{array}\right]$, the linear velocity $\left[\begin{array}{lll}\dot{x} & \dot{y} & z\end{array}\right]$ and the orientations such as the Euler angles $\left[\begin{array}{lll}\varnothing & \theta & \psi\end{array}\right]$ of the quadcopter can be obtained from the East-North-Up (ENU) reference system, whereas the angular velocity $\left[\begin{array}{lll}p & q & r\end{array}\right]$ of the quadcopter can be obtained from the local reference frame [14].

Since the quadcopter is free to rotate about the 3 axes and hence the local reference frame, a transformation is needed in order to describe the body frame relative to the ENU frame. To this end, a direction cosine matrix (DCM) is required to transform the body frame to the inertial frame or vice versa [20]. The direction cosine matrix is essentially a rotation matrix, which rotates the reference fame several times by certain amount about the specified axes. It should be noted that for a transformation from the body frame to the global frame, there are many ways of rotations to achieve the same result. In this case, we chose to use the Tait-Bryan angle $\mathrm{Z}_{1} \mathrm{Y}_{2} \mathrm{X}_{3}$ in this work as the flight controller unit [21].

According to Nelson, the rotation matrix for the combined rotations can be expressed by Eq. 1 [22].

$$
z_{\varphi} y_{\theta} x_{\emptyset}=\left[\begin{array}{ccc}
C_{\Psi} C_{\theta} & C_{\Psi} S_{\theta} S_{\varnothing}-S_{\Psi} C_{\emptyset} & C_{\Psi} S_{\theta} C_{\emptyset}+S_{\Psi} C_{\emptyset} \\
S_{\Psi} C_{\theta} & S_{\Psi} S_{\theta} S_{\emptyset}+C_{\Psi} C_{\emptyset} & S_{\Psi} S_{\theta} C_{\emptyset}-C_{\Psi} S_{\varnothing} \\
-S_{\theta} & C_{\theta} S_{\emptyset} & C_{\theta} C_{\emptyset}
\end{array}\right]
$$

where $C_{\theta}$ represents $\cos (\theta)$, and $S_{\theta}$ represents $\sin (\theta)$ and so on.

The Euler rates ( $\dot{\Psi}, \dot{\theta}$, and $\dot{\Phi})$ can be calculated from the angular velocities (p, q, and r) as shown in Eq. 2 [22]:

$$
\left[\begin{array}{c}
\dot{\Phi} \\
\dot{\theta} \\
\dot{\Psi}
\end{array}\right]=\left[\begin{array}{ccc}
1 & S_{\emptyset} \tan \theta & C_{\emptyset} \tan \theta \\
0 & C_{\varnothing} & -S_{\emptyset} \\
0 & S_{\phi} \sec \theta & C_{\phi} \sec \theta
\end{array}\right]\left[\begin{array}{l}
p \\
q \\
r
\end{array}\right]
$$

\subsection{Dynamics of quadcopter}

A quadcopter is a heavier-than-air flying machine, which generates lift via the 4 motors spinning at desired speeds. In order for the quadcopter to hover in the air, the 4 motors have to be spinning at the hovering speed. The hovering speed is a speed, at which the propellers are generating sufficient lift to overcome the gravitational pull on the quadcopter. Hence, it is intuitive that the ascending and descending motions of the quadcopter can be done by increasing or decreasing the speed of all the 4 motors concurrently by a same amount. Besides these vertical motions, the orientation of the quadcopter such as roll and pitch angles can be controlled by creating a speed difference between the pairs of motors opposite to each other. By regulating these angles, the translational motions such as the position and speed of the quadcopter can be controlled indirectly. Interestingly, a quadcopter has 1 pair of clockwise rotating and another pair of counter-clockwise rotating motors, with each pair of motors placing opposite to each other. By doing so, the torque generated when the motors spin can be cancelled out by each other when hovering or doing level flight. Hence, the yawing motion of the quadcopter can be done by allowing the 2 pairs of motors spinning at different speeds to create a net torque about the Z-axis of the quadcopter.

\section{Linear control system}

The control system used for this work is extracted from a work conducted by a group of researchers from University of Pennsylvania [13]. The control system of the quadcopter consists of 2 control loops, which are the inner and outer control loops [13]. The outer control loop functions as a position controller, which takes in the GPS data and the desired position as the inputs and uses the error between the inputs to calculate the desired attitude outputs such as roll, pitch and yaw angles, Next, these desired attitude outputs will be used together with the IMU data as the inputs for the inner control loop, which is the attitude controller. By using the attitude controller, the required changes in motors' speeds can be calculated so that the quadcopter can be controlled desirably.

The outer loop controller, which is the position controller, uses a PD controller, which can be expressed by Eq. 3 [13].

$$
\ddot{s}_{m}^{d e s}=\ddot{s}_{m, j}+k_{D, m}\left(\dot{s}_{m, j}-\dot{s}_{m}\right)+k_{P, m}\left(s_{m, j}-s_{m}\right)
$$

where $m=x_{w}, y_{w}, z_{w}$. This controller is used to calculate the desired linear acceleration, $\ddot{s}_{m}$ des , based on the difference/error between the desired velocity, $\dot{s}_{m, j}$, and actual velocity, $\dot{s}_{m}$, as well as the difference/error between desired position, $s_{m, j}$, and actual position, $s_{m}$. The constants, $k_{D, m}$ and $k_{P, m}$, are the gain parameters for the errors in the velocity and position respectively. Using the calculated desired linear acceleration, the desired attitude angle 
can be found using expressions of Eqs. 4 and 5 [13].

$$
\begin{aligned}
& \emptyset^{d e s}=\frac{1}{g}\left(\ddot{s}_{x w}{ }^{d e s} \sin \varphi_{j}-\ddot{s}_{y w}{ }^{d e s} \cos \varphi_{j}\right) \\
& \theta^{d e s}=\frac{1}{g}\left(\ddot{s}_{x w}{ }^{d e s} \cos \varphi_{j}+\ddot{s}_{y w}{ }^{d e s} \sin \varphi_{j}\right)
\end{aligned}
$$

Next, the attitude controller generates the required change in motors' speeds based on the desired attitude angle. Similar to the position controller, the attitude controller is also a PD controller. The attitude controller can be expressed by Eqs. 6, 7 and 8 [13].

$$
\begin{aligned}
& \Delta \omega_{\varnothing}=k_{p, \emptyset}\left(\emptyset^{d e s}-\emptyset_{t}\right)+k_{d, \emptyset}\left(p^{\text {des }}-p_{t}\right) \\
& \Delta \omega_{\theta}=k_{p, \theta}\left(\theta^{\text {des }}-\theta_{t}\right)+k_{d, \theta}\left(q^{\text {des }}-q_{t}\right) \\
& \Delta \omega_{\varphi}=k_{p, \varphi}\left(\varphi^{\text {des }}-\varphi_{t}\right)+k_{d, \varphi}\left(r^{\text {des }}-r_{t}\right)
\end{aligned}
$$

Finally, the desired rotational speed for each motor can be calculated using Eq. 9 [13].

$\left[\begin{array}{l}\omega_{1}{ }^{\text {des }} \\ \omega_{2}^{\text {des }} \\ \omega_{3}^{\text {des }} \\ \omega_{4}{ }^{\text {des }}\end{array}\right]=\left[\begin{array}{cccc}1 & 0 & -1 & 1 \\ 1 & 1 & 0 & -1 \\ 1 & 0 & 1 & 1 \\ 1 & -1 & 0 & -1\end{array}\right]\left[\begin{array}{c}\omega_{h}+\Delta \omega_{f} \\ \Delta \omega_{\emptyset} \\ \Delta \omega_{\theta} \\ \Delta \omega_{\varphi}\end{array}\right]$

Whereby the motor speed at a hovering state is shown in Eq. 10 [13].

$\omega_{h}=\sqrt{\frac{m g}{4 k_{F}}}$

The change in motor speed catering for the vertical motion can be expressed by Eq. 11 [13].

$\Delta \omega_{F}=\frac{m}{8 k_{p} \omega_{n}} \ddot{S}_{z w}{ }^{d e s}$

\section{Methodology}

The proposed autonomous landing system for UAVs consists of a quadcopter and a moving platform as shown in Fig. 2. Unlike the conventional autonomous landing using computer vision that uses on-board computer vision, off-board computer vision is used in this work instead. A camera, which is upward, facing the sky, captures the relative position of the quadcopter to the moving platform.

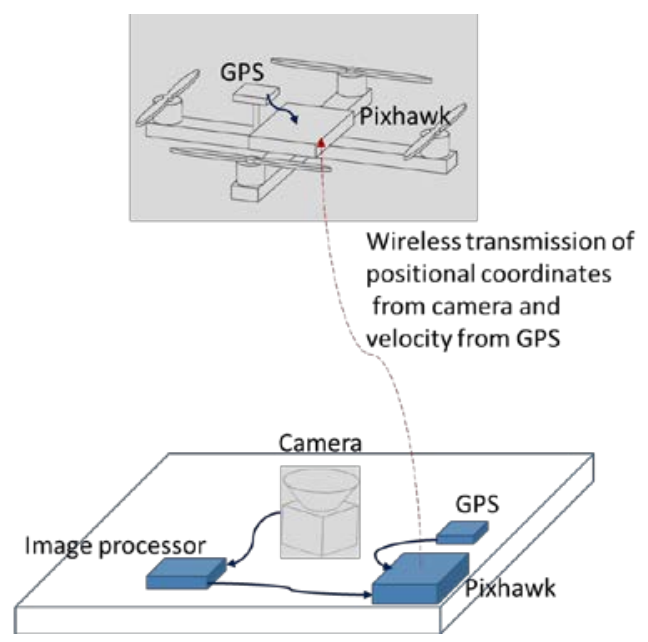

Figure 2. Setup of the vision-based landing system. 
In order to have a better estimation of the motion of the moving platform, a Pixhawk and a GPS module are used to provide the heading and the velocity of the moving platform. By doing so, the quadcopter can use the motion of the moving platform as the target, and the controller corrects the motion of the quadcopter whenever the errors in the position and velocity are detected.

The raw data captured by the camera is transferred from the image processor, which is the computer, to the quadcopter using telemetry modules. In this work, 2 pairs of Xbee telemetry modules are used. One pair of Xbee telemetry modules is used to transfer the information such as raw position of the quadcopter in the captured image, and heading and velocity of the moving platform; whereas another pair is used for debugging purposes.

\section{System implementation}

\subsection{Quadcopter}

The quadcopter used in this work is a custom-built craft made up of components and devices that can be found easily in the market. The quadcopter uses a DJI F450 frame, which has a tip-to-tip length of $45 \mathrm{~cm}$. Also, A2212/13T 1000KV brushless motors and $8 \times 4.5$ propellers are used on the quadcopter as the lifting mechanism. Besides, a Pixhawk is installed on the quadcopter to act as the flight controller unit. The Pixhawk has a built-in Inertial Navigation System (INS), which includes sensors such as accelerometer and gyroscope. Also, an Ublox neo-6m GPS module is connected to the Pixhawk to provide positional information about the quadcopter to the controller, and an XL-MaxSonar MB1240 ultrasonic rangefinder is used to give the altitude of the quadcopter. Other than that, 2 Xbee telemetry modules are connected to the Pixhawk to allow wireless communication with other devices such as a moving platform and computer.

\subsection{Moving platform}

Figure 3 shows the setup of the moving platform, which is made up of a Styrofoam board with a string tied at the front end. The platform is designed to have dimensions of $1.2 \mathrm{~m} \times 1.2 \mathrm{~m}$ to model the size of a vehicle such as a car or a small part of a ship. On the platform, a Microsoft Surface Pro tablet is placed at the center of the platform. The reason for using Surface Pro in this work is that Surface Pro has a front viewing camera that can capture decent images and Surface Pro has sufficient image processing power to serve the landing purpose. Besides, the Surface Pro tablet is thin and light, which would not obstruct the landing maneuver of the quadcopter when the tablet is placed lying flat on the platform. Besides Surface Pro, a Pixhawk is used to provide the information such as heading and provide peripheral connections to the Surface Pro and Xbee modules.

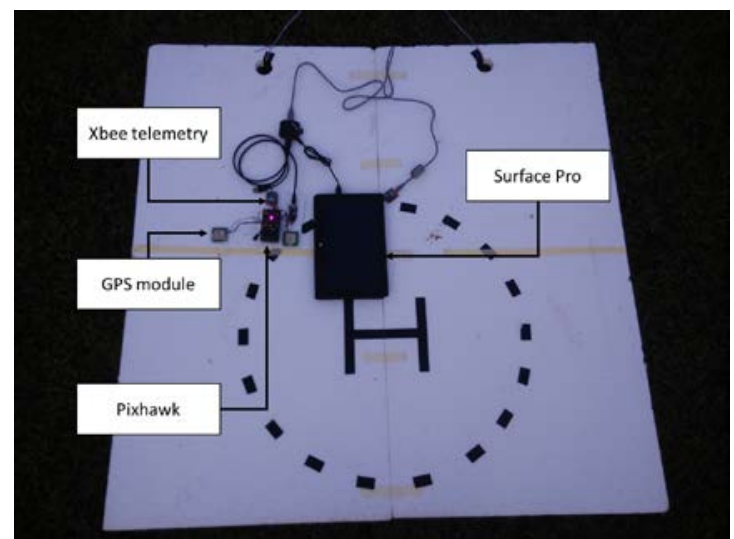

Figure 3. Equipment setup on the moving platform.

\subsection{Arducopter firmware}

The firmware loaded in the 2 Pixhawks on the quadcopter and moving platform is Arducopter Version 3.3-dev firmware $[23,24]$. The firmware is modified slightly and a set of control algorithms is added to the firmware. The quadcopter's firmware is modified in such a way that the added control code can be assessed during flight testing by switching a toggle on the RC transmitter, whereas the moving platform's firmware is modified in such a way that the Pixhawk acts as a transmission station, which transfers the data from Surface Pro to the quadcopter.

\subsection{Mavlink protocol}

The information such as the position and heading angle can be transferred between the devices using Mavlink Protocol. Mavlink Protocol allows the information to be encoded into one packet of messages, and decodes the messages when the devices receive the full packet of messages. This ensures that the messages received by the 
devices are complete without dropping any of the information. The Mavlink messages can be transferred over either using a USB or wireless telemetry module such as the Xbee module. In this work, Mavlink Protocol is used to transfer the position information from Surface Pro to Pixhawk on the moving platform via a USB cable. On the other hand, Mavlink is also used to transfer the information such as the position and heading angle from Pixhawk on the moving platform to the quadcopter via Xbee telemetry modules.

\section{Computer vision}

Computer vision is used to recognize the quadcopter and detect its relative position of the quadcopter through a set of filters, which are available in the OpenCV library [25]. Real-time image processing is performed in our application. Figure 4 shows an image captured and the result of image processing. As illustrated in the pseudo code 1 , the first filter applied to the real-time image is the grayscale filter. In our experiment, it is assumed that the landing is performed in an open field without any other tall structures such as trees, lampposts, and buildings obstructing the view. Hence, the image captured will most likely to be bright blue sky and white clouds as the background, which makes the quadcopter appear dark in the image. As such, it is reasonable to use the grayscale filter followed by a second filter, a threshold filter, to make the bright blue sky and the white clouds become white pixels and the dark quadcopter appear black in the image with a threshold level of 50. An invert filter is applied to make the quadcopter white against a black background. The next filter is the findContour filter. FindContour filter is used to draw the outline of the quadcopter in the image, and it is applied because the next filter, which is the minenclosingcircle filter, requires a contour image as the input. The minenclosingcircle filter will provide the radii and the centers of the circles detected as the output. A search function is used to find the largest circle that encloses the whole quadcopter. Finally, the radius and center coordinates of the largest circle is then sent to Pixhawk on the moving platform using Mavlink protocol through a FTDI cable.

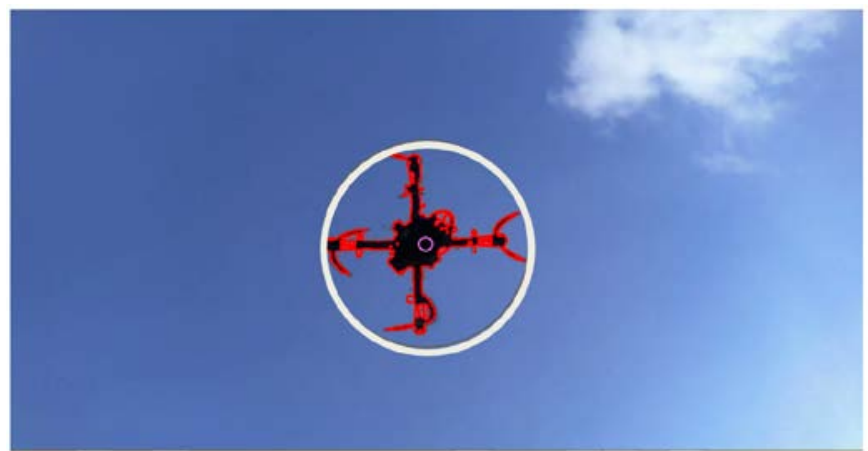

Figure 4. Determination of the size and the location of the quadcopter using the OpenCV library.

\section{Pseudo code 1.}

Get image from camera
Convert from RGB to grayscale
Apply threshold
Invert image
Find contour
Initialize arrays Center and radius
Initialize and set temp = 0
Initialize index
Get contour size
For $\mathrm{i} \rightarrow$ countour size do
Apply approxPolyDP function
Apply minenclosingcircle function
If radius[i] $>$ temp
$\quad$ Temp=radius[i]
$\quad$ Index $=\mathrm{i}$
End if
End for
If contour size != 0
Send mavlink message
End if




\section{Mathematical model for position estimation}

The relative position of the quadcopter to the moving platform can be found by measuring the relative position of the quadcopter in the image to the center of the captured images. However, a mathematical model is needed to estimate the actual position of the quadcopter in reality. As such, a mathematical model is developed to derive the actual position of the quadcopter from the captured image using simple trigonometry. The relationship between the position of the quadcopter and its image in the camera can be depicted in Fig. 5.

By referring to Fig. 5, the relationship between the physical dimensions of the quadcopter such as the tip-to-tip length, $\mathrm{L}$, the actual position relative to the camera on the moving platform, $\mathbf{D}$, to the image position, $\mathbf{P}$, and the radius of the circle enclosing the quadcopter in the image, $\mathrm{R}$, can be established as shown in Eq. 12.

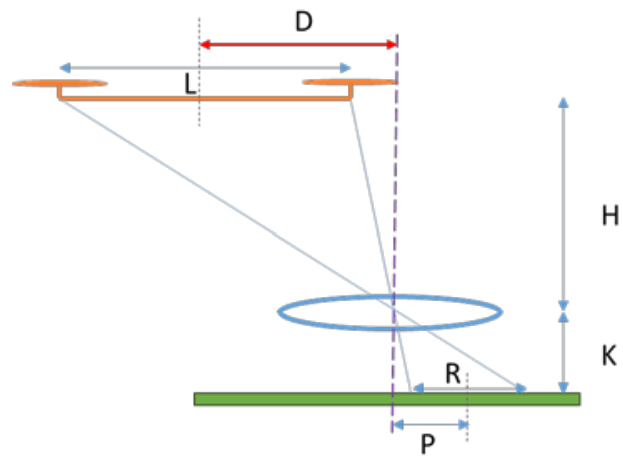

Figure 5. Graphical representation of the mathematical model.

$\frac{P_{n}}{D_{n}}=\frac{R}{L}$

where n denotes x or y, $D=\left\{\begin{array}{l}D_{x} \\ D_{y}\end{array}\right\}$, and $\boldsymbol{P}=\left\{\begin{array}{l}P_{x} \\ P_{y}\end{array}\right\}$.

In Eq. 12, it is obvious that the actual relative position of the quadcopter can be found by defining and measuring $\mathrm{P}, \mathrm{L}$ and R. Also, it is easy to understand that L can be easily obtained by measuring the physical dimensions of the quadcopter, and $\mathrm{P}$ and $\mathrm{R}$ can be obtained using the computer vision. However, it is found through experiments that the radius of the circle keeps fluctuating even through the quadcopter is hovering at a specific altitude. This might result in poor estimation of the quadcopter position in the real flight test. Hence, an ultrasonic rangefinder, which is found to be more consistent than using the radius of the circle, is used instead.

The relationship between the radius of the circle in the image and the altitude of the quadcopter can be described by Eq. 13 using trigonometry.

$$
R \propto \frac{1}{H}
$$

By allowing the quadcopter to hover at different altitudes above the camera, the relationship between the radius of the quadcopter in the image and the altitude of the quadcopter can be plotted in Fig. 6. By employing the curve fitting technique, the reciprocal relationship achieves a high $\mathrm{R}^{2}$ value of 0.9875 with the incorporation of a small offset. However, it is also important to point out that the fitted curve is specific to the camera used, which means that the fitted curve function is only applicable when the Surface Pro front camera is used.

Having found the relationship between the radius of the circle and the altitude of the quadcopter, the position of the quadcopter can be obtained using Eq. 14 obtained by rearranging Eq. 12.

$$
\boldsymbol{D}=\boldsymbol{P} \times \frac{L}{R}
$$

However, $\mathbf{D}$ is measured with respect to the reference frame of the moving platform, which is $X_{L} Y_{L}$. Therefore, a rotation matrix is needed to convert $\mathbf{D}$ to the global reference frame, which is $X_{w} Y_{w}$, because the positional controller of the quadcopter requires the position of the quadcopter with respect to the global reference frame as shown in Fig. 7. The conversion from the local reference frame to the global reference frame can be performed using Eq. 15, where $\varphi$ is the heading angle of the moving platform.

$$
\left[\begin{array}{l}
D_{w, x} \\
D_{w, y}
\end{array}\right]=\left[\begin{array}{cc}
\cos \varphi & -\sin \varphi \\
\sin \varphi & \cos \varphi
\end{array}\right]\left[\begin{array}{l}
D_{x} \\
D_{y}
\end{array}\right]
$$




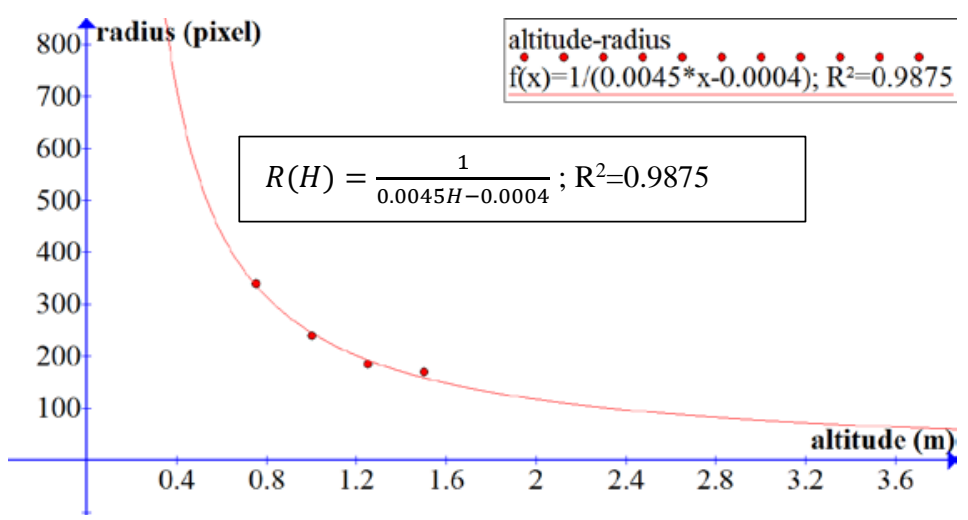

Figure 6. Graph of the radius vs the altitude.

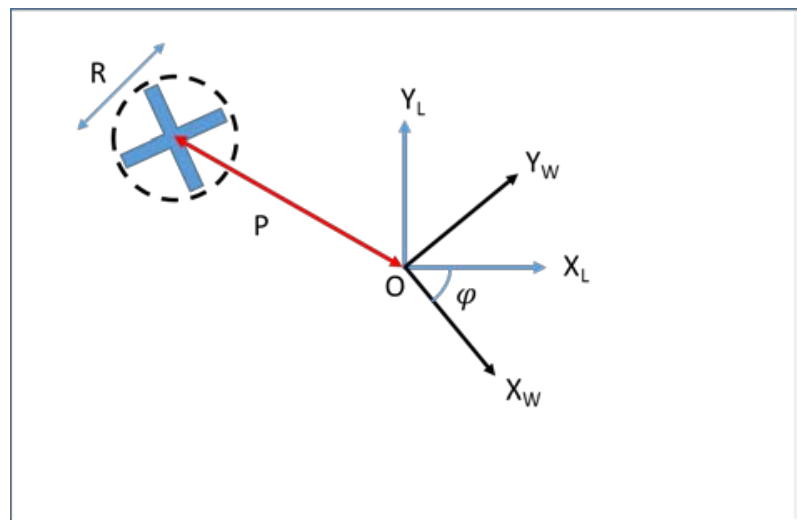

Figure 7. Graphical representation of the captured image and the reference frames.

\section{Flight tests}

In order to evaluate the effectiveness of the methodology, two tests were carried out, namely, the stationary platform test and the moving platform test.

\subsection{Stationary platform test}

In the stationary platform test, as the name implies, the platform was left stationary, and the quadcopter would then try to hover above the stationary platform and perform landing using the information obtained wirelessly from the platform. The stationary platform test served as a preliminary test to check if the controllers of the quadcopter were working correctly. The stationary landing test was conducted for 10 times and the whole landing process was recorded. The distance error of the landed quadcopter from the center was noted. After the quadcopter had successfully landed on the stationary platform, which meant that the controllers were working properly, the moving platform test was then carried out.

\subsection{Moving platform test}

In the moving platform test, the same platform was made to move by a person pulling a nylon string that was connected to the platform. The image sequence of the landing maneuver on the moving platform is shown in Fig. 8. Autonomous landings were performed at platform-moving speeds of $0.5 \mathrm{~m} / \mathrm{s}, 1 \mathrm{~m} / \mathrm{s}$ and $2 \mathrm{~m} / \mathrm{s}$. It is believed that the quadcopter can achieve autonomous landings at higher platform-moving speeds, and the $2-\mathrm{m} / \mathrm{s}$ limit was due to the fact that the moving platform was pulled by a person in this test.

\section{Results}

\subsection{Stationary platform}

Figure 9 shows the landing position of the quadcopter on the stationary platform. During the stationary test, it was observed that the quadcopter would sometimes drift away from the center of the platform, but the controllers were still able to bring it close to the center of the platform. In addition to that, it was observed that five out of ten landings fell in the good region, which was within a $30 \mathrm{~cm}$ radius from the center. Two landings fell in the moderate 
region, which was between $30 \mathrm{~cm}$ and $60 \mathrm{~cm}$. There were three landings falling beyond $60 \mathrm{~cm}$, which was considered poor. The largest deviation from the center was $84 \mathrm{~cm}$.

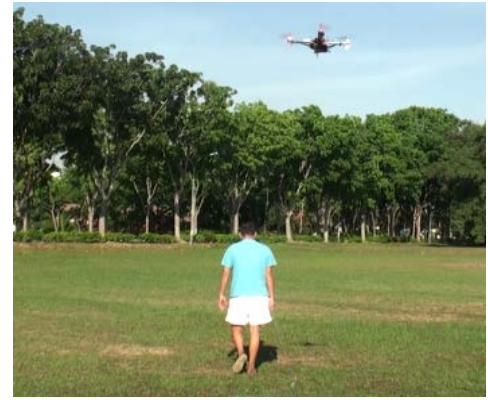

(a)

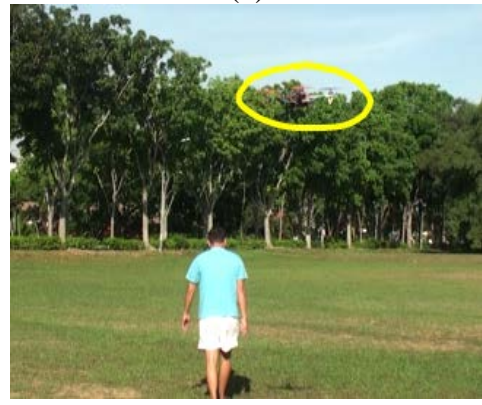

(c)

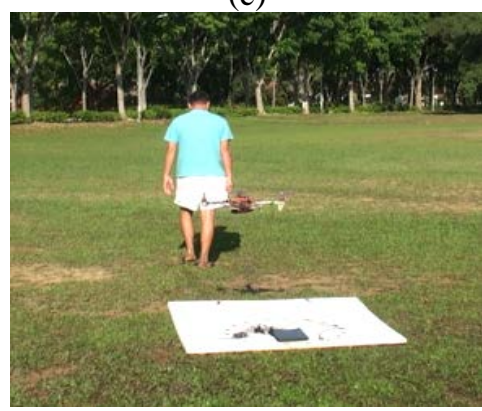

(e)

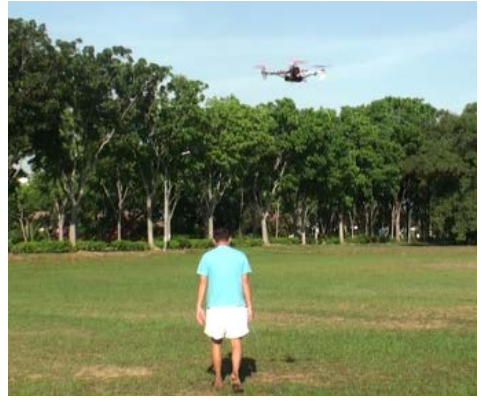

(b)

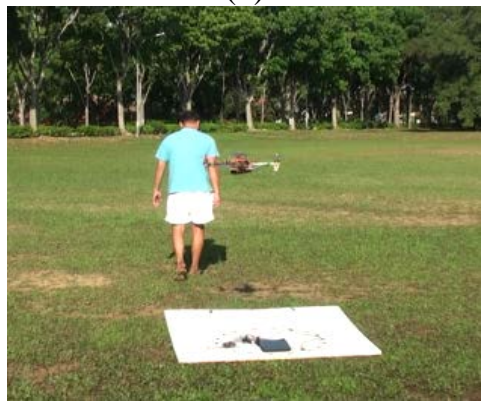

(d)

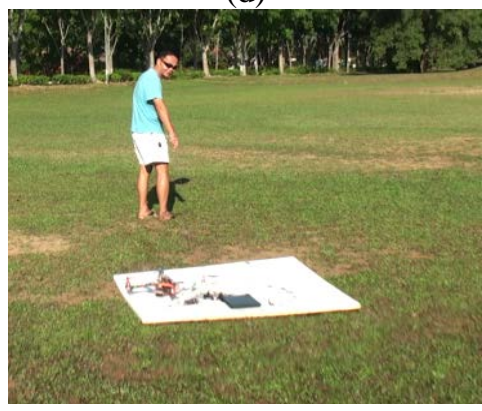

(f)

Figure 8. Image sequence of the moving platform test.

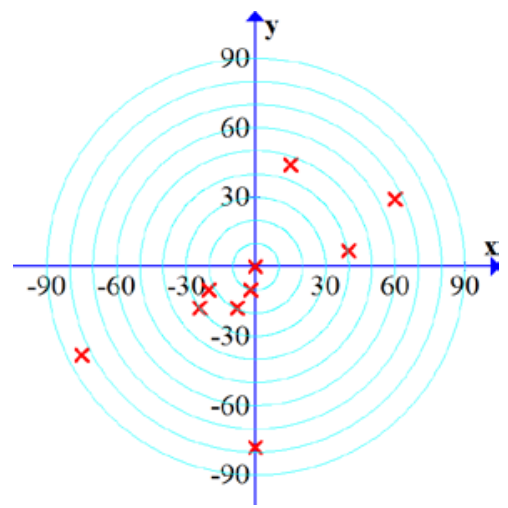

Figure 9. Landing positions (in $\mathrm{cm}$ ) on the stationary platform.

\subsection{Moving platform}

As stated earlier, the moving platform test was conducted at a platform moving speed of $0.5 \mathrm{~m} / \mathrm{s}, 1 \mathrm{~m} / \mathrm{s}$ or $2 \mathrm{~m} / \mathrm{s}$. In order to evaluate the consistency of the landing, five samples of the landing results were collected at each platform moving speed. The results of the landings at different platform moving speeds with the mean values and standard errors are shown in Fig. 10. It can be seen that the landing error increases as the platform moving speed increases. In addition to that, it is noted that the consistency of the landing is significantly higher at a lower platform moving speed. 


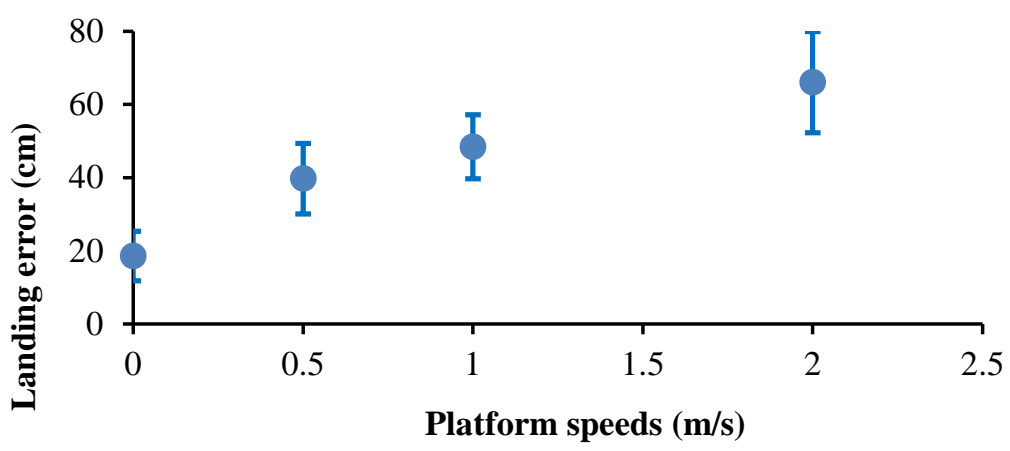

Figure 10. Landing error at various platform moving speeds.

\subsection{Discussion}

In most of the flight tests, it was observed that the quadcopter would drift in random directions occasionally when it was hovering over the platform. The slight drifting of the quadcopter was due to the fluctuation of the velocity values from the two GPS modules. It is well known that the GPS can only provide accuracy of $2.5 \mathrm{~m}$, which means that the single point position solution obtained from GPS will be scattered around the center but still within the $2.5 \mathrm{~m}$ radius. Hence, it is no doubt that the derivative of the position obtained from GPS will fluctuate.

Besides the GPS error, the weather condition [26-28] also plays an important part in the flight test outdoor. The windy condition at the test site has caused the quadcopter to be blown away, as the controller used for the quadcopter does not take into account of the wind effect. Hence, a new controller that can compensate for the wind effect should be implemented for better test results.

Also, it was found that the accuracy of the landing was within a $90-\mathrm{cm}$ radius, which was not considered high, but it was sufficient for the quadcopter to land on the platform with dimensions of $1.8 \mathrm{~m} \times 1.8 \mathrm{~m}$. Despite the deviation, the landing accuracy is still higher as compared to GPS-based landing as the normal GPS has accuracy of $2.5 \mathrm{~m}$.

Other than that, it was observed that the scope of view of the camera was not the same in all directions. The Surface Pro front camera has 16:9 aspect ratio by default and the viewing angles are measured approximately to be $60^{\circ}$ and $36^{\circ}$ corresponding to the length and the width of the camera image respectively. After conducting the flight tests, it was observed that the quadcopter often flied out of the scope of vision of the camera, and this caused the landing maneuver to be aborted. As such, it is suggested that a camera with a wide viewing angle should be used in place of the Surface Pro front camera.

\section{Conclusions}

In this article, the vision-based landing of a quadcopter on a moving platform is presented and discussed. Unlike other researches, the camera is mounted on the moving platform instead of being installed on the quadcopter. This method has an advantage in terms of weight, as the gimbal and the camera do not have to be installed on the quadcopter. This is advantageous as a lighter quadcopter means that the flight duration is longer. In the stationary platform test conducted outdoor, 5 out of 10 landings fell within $30 \mathrm{~cm}$ from the center and the poorest landing was $84 \mathrm{~cm}$ from the center. In the moving platform test conducted outdoor, the maximum platform-moving speed for autonomous landing was $2 \mathrm{~m} / \mathrm{s}$, which was limited by the running speed of the man who was pulling the moving platform while running. Hence, it is proven that this methodology is feasible. For future work, it is recommended that pattern recognition could be used instead of using a threshold filter in order to improve the recognition capability for a more complex background.

\section{Acknowledgment}

The work was supported by Nanyang Technological University under the Undergraduate Research Experience on Campus (URECA) program.

\section{References}

[1] Aviation knowledge: Unmanned aerial vehicles. http://aviationknowledge.wikidot.com/aviation:uav

[2] Zhong ZW, Ma ZX, Jayawijayaningtiyas, Ngoh JH. Visual signature reduction of unmanned aerial vehicles. In: Proceedings of SPIE Security+Defence (Vol. 9997), Conference on Target and Background Signatures II. 
Sept. 26-27, 2016. Edinburgh, Scotland. Article Number: UNSP 999707.

[3] Newcome LR. Unmanned aviation: a brief history of unmanned aerial vehicles. American Institute of Aeronautics and Astronautics; 2004.

[4] Finkelstein R. The Ubiquitous UAV. http://www.pdffiller.com/210554-The_Ubiquitous_-UAV-THE-UBIQ UITOUS-UAV---Robotic-Technology-Inc--Various-Fillable-Forms?v=sharing\&utm_expid=2952066-165. TWsjWE6xQpOHgmO31XEXfQ.1\&utm_referrer=https\%3A\%2F\%2Fwww.google.com.my\%2F

[5] Gbiansky A. Quadcopter dynamic, simulation and control. http://andrew.gibiansky.com/blog/physics/quadc opter-dynamics/

[6] Chee KY, Zhong ZW. Control, navigation and collision avoidance for an unmanned aerial vehicle. Sensors and Actuators A: Physical. 2013;190:66-76.

[7] Ollero A, Merino L. Unmanned aerial vehicles as tools for forest-fire fighting. Forest Ecology and Management. 2006;234(1):S263.

[8] Rusnell M, Gano S, Perez V, Renaud J, Batill S. Morphing UAV pareto curve shift for enhanced performance. In: 45th AIAA/ASME/ASCE/AHS/ASC Structures, Structural Dynamics \& Materials Conference; 2004. p. 1682.

[9] Liu CA, Li WJ, Wang HP. Path planning for reconnaissance UAV based on Ant Algorithm. Kongjun Gongcheng Daxue Xuebao(Ziran Kexue Ban)/ Journal of Air Force Engineering University(Natural Science Edition)(China). 2004;5(2):9-12.

[10] Zhong ZW, Yee JH. Study of optimization of air route networks and locations of crossing waypoints for SEA. In: 2018 14th IEEE/ASME International Conference on Mechatronic and Embedded Systems and Applications (MESA); 2018. p. 1-6.

[11] Mahony R, Kumar V, Corke P. Multirotor aerial vehicles: Modeling, estimation, and control of quadrotor. IEEE Robotics \& Automation Magazine. 2012;19(3):20-32.

[12] Parunak HV, Purcell M, O'Connell R. Digital pheromones for autonomous coordination of swarming UAV's. Ann Arbor (vol. 1001); 2002. p. 48105-1579.

[13] Michael N, Mellinger D, Lindsey Q, Kumar V. The grasp multiple micro-uav testbed. IEEE Robotics \& Automation Magazine. 2010;17(3):56-65.

[14] Friis J, Nielsen E, Andersen RF, Boending J, Jochumsen A, Friis A. Autonomous landing on a moving platform. Control Engineering, 8th Semester Project. Aalborg University, Denmark; 2009.

[15] Herissé B, Hamel T, Mahony R, Russotto FX. Landing a VTOL unmanned aerial vehicle on a moving platform using optical flow. IEEE Transactions on Robotics. 2012;28(1):77-89.

[16] Lange S, Sünderhauf N, Protzel P. Autonomous landing for a multirotor UAV using vision. In:SIMPAR 2008 Intl. Conf. on Simulation, Modeling and Programming for Autonomous Robots; 2008.p. 482-491.

[17] Saripalli S, Sukhatme GS. Landing on a moving target using an autonomous helicopter. In: Field and Service Robotics; 2006.p. 277-286.

[18] Lee D, Ryan T, Kim HJ. Autonomous landing of a VTOL UAV on a moving platform using image-based visual servoing. In: IEEE International Conference on Robotics and Automation (ICRA); 2012.p. 971-976.

[19] Kim J, Jung Y, Lee D, Shim DH. Outdoor autonomous landing on a moving platform for quadrotors using an omnidirectional camera. In: International Conference on Unmanned Aircraft Systems (ICUAS); 2014. p. 1243-1252.

[20] Premerlani W, Bizard P. Direction cosine matrix imu: Theory. USA: Diy Drone. 2009:13-15.

[21] Roberson RE, Schwertassek R. Dynamics of multibody systems (vol. 18). Springer-Verlag Berlin; 1988.

[22] Nelson RC. Flight stability and automatic control (vol. 2). WCB/McGraw Hill; 1998.

[23] Short J. Arducopter V3.3-dev, ed: Ardupilot. 2014.

[24] Ardupilot. APM. http://ardupilot.com/

[25] OpenCV. OpenCV API reference.http://docs.opencv.org/modules/refman.html

[26] Xie Z, Zhong ZW. Aircraft path planning under adverse weather conditions. In:MATEC Web of Conferences; 2016. p. 15001.

[27] Aneeka S, Trong N, Goh JW, Lee YX, Phyoe SM, Zhong ZW. An experimental study on the cost and capacity effects of contingency routes under adverse weather conditions within ASEAN region. In: International Conference on Education, Transportation and Disaster Management. Singapore; 2017.

[28] Lim WX, Zhong ZW. Re-planning of flight routes avoiding convective weather and the "three areas". IEEE Transactions on Intelligent Transportation Systems. 2018;19(3):868-877.

[1] C 2019 by the author(s). This work is licensed under a Creative Commons Attribution 4.0 International License (http://creativecommons.org/licenses/by/4.0/). Authors retain copyright of their work, with first publication rights granted to Tech Reviews Ltd. 